\title{
The applications of image processing technology in the analysis of the factors of shooting rate
}

\author{
Wang Zhiwen ${ }^{1, a}$, Wang Pengtao ${ }^{2, b}$,Tang Bowen ${ }^{2, \mathrm{c}}$ and Hu Zhenghuan ${ }^{2, \mathrm{~d}}$ \\ ${ }^{1}$ College of computer science and communication engineering, Guangxi University of science and \\ technology, Liuzhou, 545006, China \\ ${ }^{2}$ College of electrical and Information Engineering, Guangxi University of science and technology, \\ Liuzhou, 545006 , China

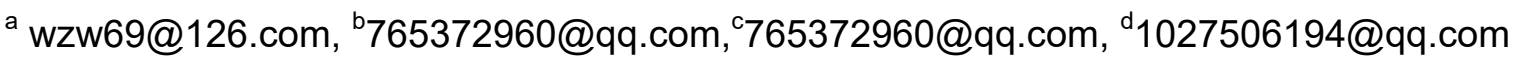

Keywords: Image processing, Shooting height, Shooting angle, Shooting speed.

\begin{abstract}
The main factors of affecting the shooting rate have the shooting angle, the shooting speed and the shooting height. Through image processing technology, we got the accurate basketball motion track. Through research and analysis the motion track with the basic theory of mechanics, we include that the shooting angle and shooting speed are affected by the shooting height when we shoot at the basketball, which provides effective theoretical basis for shooting training.
\end{abstract}

\section{Introduction}

With the popularity of digital imaging products, digital cameras are used in the various areas of our work and life, and therefore the changes about scientific instruments quickly are penetrating into all walks of life. We take advantage of this technology to turn studying objects into dynamic or static pictures, and then process the picture of the specific pixel in order to get the graphics or equations which are required for the study. Finally, we analyze and discuss the study parameters of the experiment [1].

In this experiment, we use digital cameras to get a full shot video, and turn the video images into a static picture with the help of the MATLAB. We process static image and by means of frame difference to make the image overlay, thus we get the trajectory image from a starting point to the end shot point of the basketball into the basket. In order to obtain a trajectory map, we analyze and calculate the relationship between shot angles and shot speed with basic mechanics theory from one hand shoulder shot technology in the free throw line [2], therefore we provide an effective method and reference for shooting rate.

\section{Research Methods}

Step 1: Place the camera. Experimental study of shooting in the free-throw line facing backboard, the camera sets up in a perpendicular bisector of the ring projection connection point and shoots, and we ensure that the video completely captures the flight trajectory from the leaving hand to go into the basketball hoop. So we find a suitable location to fix the cameras.

Step 2:Video capture. We select an athlete who shoots in one hand shoulder shot technology under the free throwing state and the camera shoots continuously.

Step 3: Video processing. We make the finished video shooting pass into the computer and divide video into a static picture frame by frame by MATLAB.

Step 4: Image Processing. In the completed static image segmentation, we select a group of the static images which have been completely segmented. The process is the moment from the basketball leaving hand to completely the enter the basketball ring and we synthesize basketball trajectory in real-time image by the inter-frame difference method.

The basic principle of background subtraction includes that: First, we use the pre-stored or real-time image sequence to obtain statistical background model for each pixel. We get the background model 
$B_{k}$. When the image of the current frame $f_{k}$ which contains the moving targets is subtracted from the background model .That is formula (1), where in $D_{k}$ is the subtraction result between the current frame and background model; Next, the calculation results is binarized under a certain limit of threshold value $T$, as shown in equation (2).It is judged that those pixels which are current large deviation values from the background model are the moving target pixels, which $R_{k}$ contain binaryzation target images. Due to there are differences in gray scale or color between the moving object and the background. After the operation of subtraction and threshold, we will directly get the result which include the target location, size, shape, etc, therefore we obtain the complete target information [3] [4].

$$
\begin{aligned}
& D_{k}(x, y)=\left|f_{k}(x, y)-B_{k}(x, y)\right| \\
& R_{k}(x, y)=\left\{\begin{array}{l}
1, D_{k}(x, y)>T \\
0, \text { else }
\end{array}\right.
\end{aligned}
$$

In the paper, we choose the2632th frame shot as a background image, shown in Fig.1.The video fps is 30 frames per second. In order to ensure that the basketball are non-overlapping in synthesize the trajectory chart, we choose the 2634th frame image as the next one, as shown in Fig. 2.We will make the 2632th and 2634th synthesize as a new picture, shown in Fig. 3. We can synthesize the entire group of pictures in the same method, we can get the entire process track from the shot to the basketball into the hoop, shown in Fig. 4.

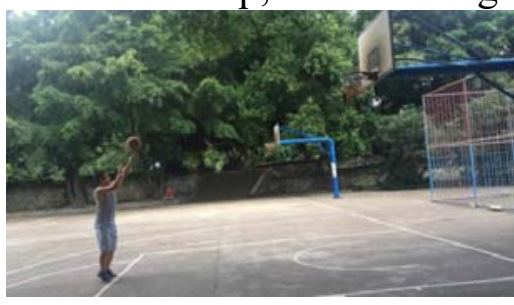

Fig. 1. The 2632th frame

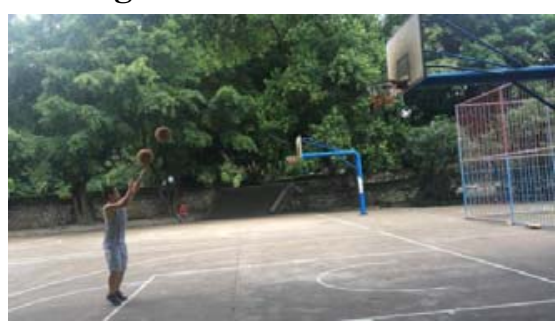

Fig. 3. The synthesis picture through the 2632th frame and the 2634th frame

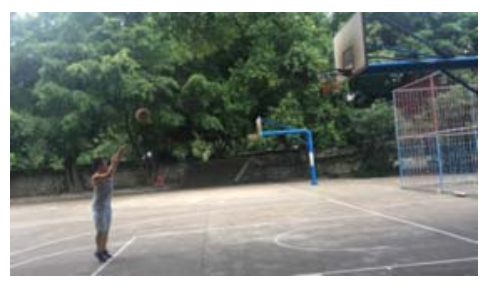

Fig. 2. The 2634th frame

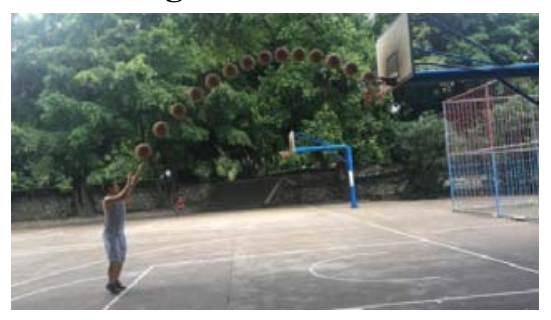

Fig. 4. Entire basketball real-time flight synthesis trajectory

\section{Experiment Results Analysis}

From the perspective of physics shooting technique, we usually regard basketball as a particle. In the Fig. 4, we can easily find the basketball center and connect each basketball center, so that we get the basketball flight trajectory, as shown in Fig.5.

According to the above method, you can get every shot trajectory, and then we establish several trajectories in the same coordinate system for observation and analysis, Fig. 6 is repeatedly shooting the basketball trajectories which are basketball's real trajectory.Track1 misses and pop out along the back porch after hitting the basket. Track 2 hits and approximately enter along the rim into the basket. Track 3 hits, and the approximately enter along center of the basket. Track 4 also hits, and approximately along frontier enter into the basket attached to the basket. Track 2,3,4,are almost the same shot, but track 1 leaving hand point is higher than the shot 2,3,4.

By the analysis of the four tracks, when the basketball hits, the horizontal movement distance of track 2 is longer than the track 3 and track 4 and three tracks are 16 pictures.Through the same basketball's movement time, we get the track2's horizontal velocity is faster than the track 3and track 
4. Therefore we include that when a certain shot height, the greater the leaving hand speed, the greater the angle of the shot.

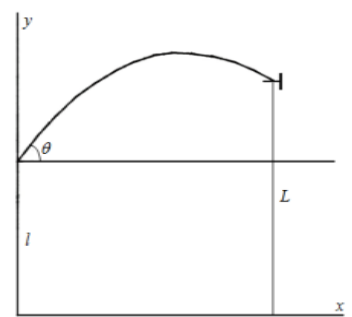

Fig. 5. Basketball flight trajectory

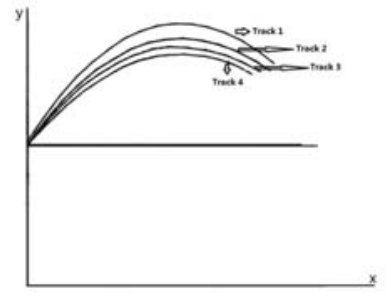

Fig.6. multiple shooting's basketball motion track

Without considering air resistance. In this paper, we fix the shooting distance in the experiment , and the same player shoot in free throw line with one hand shoulder shot technology, we keep the same throwing height when throw the basketball. Set shooting distance is, the ring height above the ground is, shot height of leaving hand, the vertical displacement from leaving hand to enter into the basket is , set release speed , shot angle, according to the picture tracks can be obtained[5,6,7]:

In the horizontal direction

$$
\left\{\begin{array}{c}
V_{x}=V \cdot \cos \theta \\
x=V \cdot \cos \theta \cdot t
\end{array}\right.
$$

In the vertical direction

$$
\left\{\begin{array}{c}
V_{y}=V \cdot \sin \theta \\
y=V \cdot \sin \theta \cdot t-\frac{1}{2} g t^{2}
\end{array}\right.
$$

Elimination parameters $t$, we can get $V$ by Equ.(5).

$$
V^{2}=\frac{g x^{2}}{2 x \sin \theta \cos \theta-2 y \cos ^{2} \theta}=\frac{g x^{2}}{x \sin 2 \theta-y \cos 2 \theta-y}
$$

In fact, we should consider to save force, it is necessary to take the minimum speed, we can get the minimum $V$ when the denominator is maximum for Equ.(5).

$$
f(\theta)=\sqrt{x^{2}+y^{2}}\left(\sin 2 \theta \frac{x}{\sqrt{x^{2}+y^{2}}}-\cos 2 \theta \frac{y}{\sqrt{x^{2}+y^{2}}}\right)-y
$$

Set the angle by Equ.(7).

$$
\sin \varphi=\frac{y}{\sqrt{x^{2}+y^{2}}}, \cos \varphi=\frac{x}{\sqrt{x^{2}+y^{2}}}
$$

Put Equ. (7) into Equ. (6), we get Equ.(8).

$$
f(\theta)=\sqrt{x^{2}+y^{2}} \sin (2 \theta-\varphi)-y
$$

From Equ. (8), we can get, if we want $\sin (2 \theta-\varphi)$ to be the maximum value, that is $\sin (2 \theta-\varphi)=1$

, when $2 \theta-\varphi=\frac{\pi}{2}$, has the minimum value, at this point $\theta=\frac{\pi}{4}+\frac{\arcsin \frac{y}{\sqrt{x^{2}+y^{2}}}}{2}$ the shooti ng is accurate and efficient. 
In the experiment, we use 30 frames / sec video camera and it means that can scan 30 pictures per second, when synthesis basketball flight trajectory, it exists a frame Interval between the previous picture and the next picture, namely, the adjacent time interval is in Fig. 4. We can calculate the time that basketball flight to the highest point through the number of balls in the picture, setting the time of reaching the highest point is, the total time from the leaving hand to enter into basketball hoop is .

According to kinematic equations, we can get Equ. (9).

$$
\tan \theta=\frac{V_{y}}{V_{x}}=\frac{g d D}{x}
$$

Shooting initial velocity can be defined by Equ. (10).

$$
V=\frac{x}{D \cos \theta}
$$

Taking the size of the basketball and hoop into account, when the basketball enters into the hoop, basketball center and the ring center must be in the same horizontal plane. The basketball can be attached to the forefront ring in the nearest distance and along the inner ring of back edge in the furthest distance, as shown in Fig. 7 and Fig. 8.
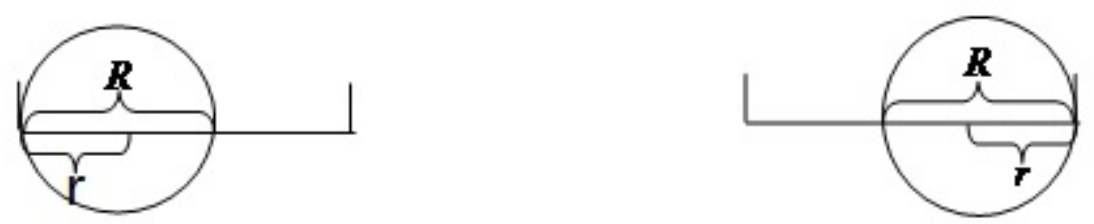

\section{Fig.7. Basket enters into the forefront Fig. 8. Basket enters into the back edge ring figure ring}

In this case, shot speed and shot angle range can be calculated by Equ.(11) and Equ.(12).

$$
\begin{gathered}
\frac{g d_{1} D_{1}}{x-R+r}<\tan \theta<\frac{g d_{2} D_{2}}{x+R-r} \\
\frac{x-R+r}{D_{1} \cos \theta_{1}}<V<\frac{x+R-r}{D_{2} \cos \theta_{2}}
\end{gathered}
$$

In the above formula, the ring radius $R$, a radius of basketball $r ; d_{1}$ and $d_{2}$ are the flight time from the highest point to forefront and rear edge ring, $D_{1}$ and $D_{2}$ are the total time in the both different flight ways.

Consider Air Resistance. According to the actual situation and movement characteristics, we only need to consider horizontal air resistance, and the resistance is proportional to the speed, scale factor $k$. Getting the horizontal differential by Equ.(13).

$$
x^{\prime}+k x=0, x(0)=0, x^{\prime}(0)=v \cos \theta
$$

For the Equ. (13), we solve the differential equations and get the result by Equ.(14).

$$
x(t)=v \cos \theta \frac{1-e^{-k t}}{k}
$$

Setting air resistance coefficient $k=0.05(1 / \mathrm{s})$ [5], the time $t<1$, and therefore air resistance and time is very small, so the formula (14) according to the Taylor expansion, more than second-order can be ignored, so we can get the distance of $x$ and $y$ by Equ.(15).

$$
\left\{\begin{array}{l}
x(t)=v \cos \theta t-\frac{k v \cos \theta t^{2}}{2} \\
y(t)=v \sin \theta t-\frac{g t^{2}}{2}
\end{array}\right.
$$

Solutions can be obtained from Equ.(15).

$$
t=\frac{2 v x \sin \theta-2 v y \cos \theta}{x g-k v y \cos \theta}
$$

Putting the formula (16) into (15), we can obtain the relationship between $x$ and $y$. 


$$
\begin{aligned}
& v^{2} y^{2} \cos \theta\left(2 g \cos \theta-k^{2} y-2 k v \cos \theta \sin \theta\right)+x^{2} g^{2} y \\
& +2 v x y \cos \theta\left(k v \cos ^{2} \theta+g v \sin \theta-k y g-2 g x v \sin \theta\right)=0
\end{aligned}
$$

Then putting $y=L-l$ into the formula (17) we can obtain relationship between shot speed and shot angle.

\section{Conclusion}

In this paper, through extract basketball movement, and analyzes the influence of shot angle and shot speed for the shoot accuracy at a fixed distance, the main conclusions are as follows:

1) With the increasing height of leaving hand, the best shot angle is reduced.

2) With the increasing height of leaving hand, the minimum release angle and its corresponding minimum release speed are reduced.

3) When the shot height is invariability, the greater shot speed, the greater angle of the shot.

4) In both cases, leaving hand speed and height are fixed, when we consider resistance, shot angle will be small.

After getting the athletes shot trajectory, we effectively use the time and distance intervals to calculate shot angle and shot speed when shooting athlete. We statistics and analysis the shot hit angle and speed .Through scientific analysis of the theory, it can provide a favorable guidance for Athletes' shooting practice to find the best training in the shooting angle they will develop fixed habits through long-term training for improving shooting.

\section{Acknowledgements}

The authors are very grateful for the support provided by the National Natural Science Foundation of China (61462008), 2015 Innovation Team Project of Guangxi University of Science and Technology(gxkjdx201504), Scientific Research and Technology Development Project of Liuzhou(2016C050205).

\section{References}

[1] Zhiwen WANG, Shaozi LI, Yanping LV, Kaitao YANG. Remote Sensing Image Enhancement Based on Orthogonal Wavelet Transformation Analysis and Pseudo-color Processing, International Journal of Computational Intelligence Systems, vol.3, no.6,pp.745-753(2010).

[2] Dunhai Wang. Study the impact of One hand shoulder shot for the shooting rate. Stationery and technology.5,167(2014).

[3] Zhiwen WANG, Shaozi LI. Adaptive Image De-noising Using Multivariate Statistical Model of Fractal-wavelet Encodes, Information, An International Interdisciplinary Journal, vol.15, no.1, pp.41-49 (2012).

[4] HonwenLin,Dan Tu,Guohui Li, The .Moving object detection method based on the statistical background model. Computer Engineering,vol.29,no.16, pp.97-99 (2003).

[5] Wugang Li,Binling Zhang. The measurements for the air resistance coefficient of the basketball based on IT. Physics and Engineering, Vol.22, pp.3: 31-33 (2012).

[6] Zhiwen WANG, Shaozi LI. Face Recognition Using Skin color Segmentation and Template Matching Algorithms, Information Technology Journal, vol.10,no.12,pp. 2308-2314(2011).

[7] Robert Collins, Alan J Lipton. A System for Video Surveillance and Monitoring. http:/www.cs.emu.edu. 